\title{
Deciding whether a law is constitutional, interpretable, or unconstitutional
}

\author{
Pablo Amorós - Ricardo Martínez • \\ Bernardo Moreno - M. Socorro Puy
}

Received: 3 November 2010 / Accepted: 18 January 2011 / Published online: 8 February 2011

(C) The Author(s) 2011. This article is published with open access at SpringerLink.com

\begin{abstract}
A high court has to decide whether a law is constitutional, unconstitutional, or interpretable. The voting system is runoff. Runoff voting systems can be interpreted both, as social choice functions or as mechanisms. It is known that, for universal domains of preferences, runoff voting systems have several drawbacks as social choice functions. Although in our setting the preferences are restricted to be singlepeaked over three alternatives, these problems persist. Runoff mechanisms are not well-behaved either: they do not implement any Condorcet consistent social choice function in undominated subgame perfect Nash equilibria. We show, however, that some Condorcet consistent social choice functions can be implemented in dominant strategies via other simple and natural mechanisms.
\end{abstract}

Keywords Runoff voting system - Condorcet consistency · Strategy-proofness · Implementation theory

JEL Classification $\quad \mathrm{C} 72 \cdot \mathrm{D} 71 \cdot \mathrm{D} 78$

\section{Introduction}

A constitutional court is a high court that verifies the constitutionality of legislation. The judgments of the constitutional court consist of selecting one among the following three alternatives: "constitutional" (the law fits in the constitution as it is written), "interpretable" (the law is accepted as soon as it is interpreted in the proper manner), and "unconstitutional" (the law does not fit in the constitution). In this setting, it is

P. Amorós $(\varangle) \cdot$ R. Martínez · B. Moreno · M. S. Puy

Departamento de Teoría e Historia Económica, Universidad de Málaga,

Campus El Ejido, 29013 Málaga, Spain

e-mail:pag@uma.es 
natural to think that the judges members of the court have single-peaked preferences over the three alternatives, as the alternative "interpretable" cannot be bottom ranked (because "unconstitutional" is to the opposite of "constitutional"). ${ }^{1}$

Normally, as a result of a consensus process, the alternative chosen by the constitutional court has the support of an absolute majority of the judges. In some situations, however, judges are unable to reach a consensus. The voting rule planned for these situations is a runoff voting system: if no alternative wins a strict majority of votes in a first round, the judges vote in a second round but only over the two alternatives that previously got the highest support. Thus, in Spain, Articles 255 and 263 of the "organic law of the judicial power" describe the voting procedure that shall govern courts. In essence, these articles pursuit the majoritarian approval. According to Article 255 , judicial decrees and sentences should be approved by a majority of votes. The judicial procedures do not admit abstention or blank vote. When a majority is not achieved, Article 263 opt for a resolution of the conflict. Essentially, a second voting round is held between the two positions with higher support in the previous voting round. Similar voting procedures govern courts in countries like USA or Germany.

In this paper, we study whether the proposed runoff voting systems are successful in selecting the alternatives that have more support among the judges, and we analyze the extent to which other voting systems can be used to achieve this goal. In order to carry out this analysis we use the principle of Condorcet consistency. This principle requires selecting an alternative that cannot be defeated by majority voting in pairwise comparisons by any other alternative. Such alternatives are known as Condorcet winners, and the fact that judges' preferences are single-peaked guarantees their existence (furthermore, for some preference profiles, the Condorcet winner may not be unique). We would like the alternative selected by the judges to be a Condorcet winner.

We may interpret runoff voting systems in two different ways, as social choice functions or as a mechanisms. The first approach consists of selecting, for each possible preference profile, the alternative that would result in the runoff voting system if all judges voted sincerely. It is known that, for preference domains larger than the one analyzed in this paper, the runoff social choice functions have several drawbacks. ${ }^{2}$ We show that many of these problems remain in our domain of single-peaked preferences over three alternatives. We prove that runoff social choice functions are not Condorcet consistent; i.e., if all judges vote sincerely, the alternative selected by the runoff voting system is sometimes such that a majority of the judges prefer the same different alternative (Proposition 1). We also show that runoff social choice functions do not satisfy strategy-proofness; i.e., they are manipulable in the sense that there exist situations in which a judge would benefit from reporting its preferences insincerely (Proposition 2). ${ }^{3}$ Finally, we show that the runoff social choice functions

\footnotetext{
1 A law might be "interpreted" in different ways. At a first stage, however, the constitutional court only has to decide whether the law is constitutional, interpretable, or unconstitutional. In the case the law is declared interpretable, a new stage will start to determine the precise interpretation. In the present paper we do not study this last stage.

2 The reader is referred to Grofman (2008) for an extensive exposition on runoff methods, their advantages and disadvantages. See also Fishburn and Brams (1981).

3 See Barberà (2010) for a complete survey on strategy-proofness.
} 
do not satisfy Maskin-monotonicity; i.e., an alternative that was selected for some preference profile may disappear from the social choice for another preference profile even though no alternative has risen in any agent's preference ranking with respect to it (Propositions 3). ${ }^{4}$

The second approach is to analyze runoff voting systems from the point of view of the implementation theory. In this case, a runoff voting system is interpreted as an extensive form mechanism in which judges make choices sequentially. Since these mechanisms induce dynamic games of complete information, we consider subgame perfect Nash equilibria. We show that, even if we focus on undominated equilibria, some alternatives which are not Condorcet winners at some preference profiles can be supported as equilibrium outcomes of the runoff mechanisms. In other words, runoff voting systems are poor mechanisms since they do not implement Condorcet consistent social choice functions in undominated subgame perfect equilibrium (Proposition 4). Bag et al. (2009) show a similar result when the domain of preferences is unrestricted. The present paper illustrates that the problem persists even when we restrict to single-peaked preferences, emphasizing the deficiencies of the runoff voting systems. Our finding, however, does not imply that Condorcet consistent social choice functions are not implementable. We show that other simple voting systems can be used to achieve this goal. In particular, we prove that some Condorcet consistent social choice functions can be implemented in dominant strategies via some simple mechanisms in which each judge only has to announce his most preferred alternative and the median of these announcements (or a careful selection from the medians, if there are more than one) is selected (Proposition 5). This is an additional argument against runoff mechanisms since, in general, implementation in dominant strategies is a much more difficult task than implementation in undominated subgame perfect Nash equilibrium. Our last result is not obvious since, in fact, not every Condorcet consistent social choice function is implementable in dominant strategies.

The paper is organized as follows: Sect. 2 provides definitions, Sect. 3 states the results, and Sect. 4 concludes.

\section{The model}

Let $N=\{1,2, \ldots, n\}$ be a set of $n \geq 3$ agents who have to jointly decide whether a law is constitutional (alternative $a$ ), interpretable (alternative $b$ ), or unconstitutional (alternative $c$ ). Let $X=\{a, b, c\}$ be the ordered set of alternatives (the order is $a>b>c$ ). General elements of $N$ are denoted by $i, j$, etc., and general elements of $X$ are denoted by $x, y$, etc.

A strict preference relation for agent $i, P_{i}$, is single-peaked if there is an alternative, called peak and denoted by $p\left(P_{i}\right)$, such that, if $y<x<p\left(P_{i}\right)$ or $p\left(P_{i}\right)>x>y$, then $x P_{i} y$. Each agent $i \in N$ has a strict and single-peaked preference relation, $P_{i}$, defined over $X$. For this case, this amounts to say that the admissible preferences for $i$ over $X$ are those represented in Table 1 (higher alternatives in the table are preferred to lower alternatives).

4 See Maskin (1999). 
Table 1 Admissible preferences for the agents

\begin{tabular}{llll}
\hline$P^{a}$ & $P^{b a}$ & $P^{b c}$ & $P^{c}$ \\
\hline$a$ & $b$ & $b$ & $c$ \\
$b$ & $a$ & $c$ & $b$ \\
$c$ & $c$ & $a$ & $a$ \\
\hline
\end{tabular}

In other words, we suppose that if the most preferred alternative for an agent is to declare the law constitutional, then to declare the law unconstitutional is his less preferred alternative, and vice versa. Let $\mathbb{P}=\left\{P^{a}, P^{b a}, P^{b c}, P^{c}\right\}$ be the set admissible preference relations, and let $P \equiv\left(P_{i}\right)_{i \in N} \in \mathbb{P}^{n}$ be a preference profile for agents in $N$. A social choice function (SCF) is a function $f: \mathbb{P}^{n} \rightarrow X$ that, for each profile $P \in \mathbb{P}^{n}$, selects one and only one alternative $f(P) \in X$. A SCF represents the objectives of a social planner.

In this paper, we consider SCFs that satisfy Condorcet consistency. For a given preference profile $P \in \mathbb{P}^{n}$ and alternatives $x, y \in X$, let $S(P ; x, y)$ be the number of agents who prefer alternative $x$ to alternative $y$ in the profile $P$; i.e., $S(P ; x, y)=$ $\#\left\{i \in N: x P_{i} y\right\}$. An alternative $x$ is a Condorcet winner at the preference profile $P$ iff $S(P ; x, y) \geq S(P ; y, x)$ for all $y \in X \backslash\{x\}$. That is, an alternative is a Condorcet winner if it is not defeated by any other alternative in majority comparisons. Although in general a Condorcet winner may not exist, once we restrict to single-peaked preferences, it does. Furthermore, the Condorcet winner may not be unique. ${ }^{5}$

Definition 1 A SCF $f$ is Condorcet consistent if it only chooses Condorcet winners; i.e., for all $P \in \mathbb{P}^{n}, f(P)$ is a Condorcet winner.

In many situations the social planner cannot directly achieve the outcomes recommended by the SCF $f$ (for instance, he may be unaware of the preferences of the agents). To obtain the alternatives prescribed by $f$ in a decentralized way, the social planner must design a mechanism which implements it. A normal form mechanism is a pair $\Gamma \equiv(M, g)$, where $M=\times_{i \in N} M_{i}, M_{i}$ is a message or strategy set for agent $i$, and $g: M \rightarrow X$ is an outcome function. A normal form mechanism induces a different game for each preference profile $P \in \mathbb{P}^{n}$. Let $(\Gamma, P)$ denote the game induced by the normal form mechanism $\Gamma$ at $P$. Let $E$ be a game theoretic solution concept (for instance, dominant strategies or Nash equilibrium). ${ }^{6}$ Given the game $(\Gamma, P)$, let $E(\Gamma, P)$ be the set of strategy profiles that are $E$-equilibrium in the game $(\Gamma, P)$.

Definition 2 A normal form mechanism $\Gamma \equiv(M, g)$ implements a SCF $f$ in $E$ equilibrium if, for each $P \in \mathbb{P}^{n}$ :

(i) there exists $m \in E(\Gamma, P)$ such that $g(m)=f(P)$, and

\footnotetext{
5 For example, if $n$ is even, the preferences of $\frac{n}{2}$ agents are $P^{a}$, and the preferences of $\frac{n}{2}$ agents are $P^{c}$, then $a, b$, and $c$ are Condorcet winners.

6 The strategy profile $m \in M$ is a dominant strategy equilibrium of $\Gamma \equiv(M, g)$ at $P \in \mathbb{P}^{n}$ if, for all $i \in N, \hat{m}_{i} \in M_{i}$, and $\hat{m}_{-i} \in M_{-i}, g\left(m_{i}, \hat{m}_{-i}\right) R_{i} g\left(\hat{m}_{i}, \hat{m}_{-i}\right)$ (where $R_{i}$ means "at least as preferred as"; in particular, if $g\left(m_{i}, \hat{m}_{-i}\right)=g\left(\hat{m}_{i}, \hat{m}_{-i}\right)$ then $i$ is indifferent between $g\left(m_{i}, \hat{m}_{-i}\right)$ and $\left.g\left(\hat{m}_{i}, \hat{m}_{-i}\right)\right)$. Similarly, $m \in M$ is a Nash equilibrium of $\Gamma$ at $P$ if for all $i \in N$ and $\hat{m}_{i} \in M_{i}, g\left(m_{i}, m_{-i}\right) R_{i} g\left(\hat{m}_{i}, m_{-i}\right)$.
} 
(ii) if $m \in M$ is such that $g(m) \neq f(P)$, then $m \notin E(\Gamma, P)$.

If such a mechanism exists, then $f$ is $E$-implementable.

An extensive form mechanism is a dynamic mechanism in which agents make choices sequentially. Given a game theoretic equilibrium concept, Definition 2 is naturally extended to deal with implementation via extensive form mechanisms. ${ }^{7}$

Next, we define two properties that are related to the implementability of a SCF.

Definition 3 A SCF $f$ satisfies strategy-proof iff, for all $P \in \mathbb{P}^{n}, i \in N$, and $\hat{P}_{i} \in \mathbb{P}$, $f(P) P_{i} f\left(\hat{P}_{i}, P_{-i}\right)$.

Roughly speaking, a SCF is strategy-proof if, in the direct mechanism associated with it, truth-telling is a dominant strategy. ${ }^{8}$ The revelation principle (Gibbard 1973) states that strategy-proofness is a necessary condition for implementation in dominant strategies.

Definition 4 A SCF $f$ satisfies Maskin-monotonicity when, for all $P, \hat{P} \in \mathbb{P}^{n}$, if $f(P) \neq f(\hat{P})$ then there exist some $i \in N$ and $x \in X$ such that $f(P) P_{i} x$ and $x \hat{P}_{i}$ $f(P)$.

Maskin-monotonicity says that if an alternative is selected by $f$ at a given preference profile $P$, then it must be also selected for any other preference profile $\hat{P}$ where no alternative has risen in any agent's preference ranking with respect to it. Maskin-monotonicity is a necessary condition for the Nash implementability of a SCF (Maskin 1999). Furthermore, it is a desirable property in itself that can be justified from a normative point of view.

Consider the runoff voting system as the one used by constitutional courts to decide whether a law is constitutional, interpretable or unconstitutional. ${ }^{9}$

Definition 5 A runoff voting system is a sequential voting system that chooses one and only one alternative and consists of (at least) two potential rounds:

Round 1: Each agent casts a vote for one alternative. If an alternative wins with a strict majority of votes, it is chosen and the voting ends. If no alternative wins with a strict majority of votes and two alternatives win more votes than the other, then go to Round 2. Ties are broken by a deterministic tie-breaking rule. ${ }^{10}$

Round 2: The two alternatives winning most votes in Round 1 face off against each other. If there is not a tie, the alternative that wins more votes is chosen and the voting ends. Ties are broken by a deterministic tie-breaking rule. ${ }^{11}$

\footnotetext{
7 To provide a detailed explanation on this approach would leave us very far, and it is beyond the purpose of this paper.

8 The direct mechanism associated with a SCF is a normal form mechanism where the message set of each agent is the set of possible preference relations and the outcome function is the SCF.

9 Moulin (1988) refers to this concept as plurality with runoff.

10 For example, if $n=10$, and the votes obtained by $a, b$, and $c$ are 4, 3, and 3, there not exist "two alternatives winning most votes in Round 1". Recently, the solution proposed by the President of the Constitutional Court of Spain for these situations is that the judges vote to break the tie before going to Round 2.

11 In the case of the Constitutional Court of Spain, these ties are broken by the casting vote of the President.
} 
There is a whole family of runoff voting systems that differ from each other in the tie-breaking rules, but have in common the skeleton of Definition 5 . Let $\Re$ denote the family of all runoff voting systems, with typical element $r$.

Observe that a runoff voting system $r \in \Re$ can be interpreted both, as a SCF or as a mechanism.

\section{Runoff voting systems as SCFs}

This approach consists of selecting, for each preference profile $P \in \mathbb{P}^{n}$, that alternative that would result in $r$ if all agents voted sincerely. More formally, for each runoff voting system $r \in \Re$ and each preference profile $P \in \mathbb{P}^{n}$, let $r(P) \in X$ be the alternative selected by $r$ at $P$ if all agents voted sincerely. Then, we can define the runoff SCF associated with $\mathbf{r}$ as the function $f^{r}: \mathbb{P}^{n} \rightarrow X$ such that, for each $P \in \mathbb{P}^{n}, f^{r}(P)=r(P)$.

Example 1 Let $n=10$. Consider a preference profile $P \in \mathbb{P}^{n}$ such that there are four voters with preferences $P^{a}$, two voters with preferences $P^{b a}$, and four voters with preferences $P^{c}$. Any runoff voting system $r \in \Re$ is such that, if agents vote sincerely in Stage 1, four agents vote for $a$, two agents vote for $b$ and four agents vote for $c$. Then we go to Stage 2, where only alternatives $a$ and $c$ run. If agents vote sincerely in Stage 2 , then six agents vote for $a$ and four agents vote for $c$. Hence, the SCF associated with any $r \in \Re$ is such that $f^{r}(P)=a$.

\section{Runoff voting systems as mechanisms}

Alternatively to the previous approach, a runoff voting system can be interpreted as a mechanism in which agents make choices sequentially. According to this interpretation, the mechanism induced by a runoff voting system (runoff mechanism, hereafter) is an extensive form mechanism that consists of, at least, the two following stages:

Stage 1: Agents simultaneously vote for one of the alternatives.

1.1 If there is one alternative $x$ that receives more than $50 \%$ of votes, then $x$ is chosen. STOP.

1.2 If no alternative receives more than $50 \%$ of votes and two of the alternatives, $x$ and $y$, receive more votes than the other, $z$, then proceed to Stage 2.

Stage 2. Alternatives $x$ and $y$ of Stage 1.2 are faced off against each other. Agents simultaneously vote for one of the two alternatives. If there is not a tie, the alternative that receives more votes is chosen. STOP.

Stages 1 and 2 do not cover all possible contingencies. They make up the common structure of all runoff mechanisms. The cases that are not covered by Stages 1 and 2 will differ depending of the particular runoff voting system considered (different runoff voting systems will induce different mechanisms). Let $\mathbb{G}(\Re)$ denote the family of all runoff mechanisms, with typical element $G \in \mathbb{G}(\Re)$.

In the next section we analyze the properties of the runoff voting systems in their twofold interpretation: runoff SCFs and runoff mechanisms. 


\section{The results}

\subsection{Runoff SCFs}

We first focus on the properties of the runoff SCFs. It is known that, for preference domains larger than the one analyzed in the present paper, the SCFs defined in this way have several drawbacks. We show that, unfortunately, many of these problems remain even when we consider our simple domain, with only three alternatives and single-peaked preferences. We highlight three of these drawbacks. First, we show that runoff SCFs do not always select the Condorcet winner.

Proposition 1 There is no runoff voting system $r \in \Re$ such that $f^{r}$ is Condorcet consistent.

Proof Consider Example 1 of the previous section. Alternative $b$ is the only Condorcet winner at $P$ (an absolute majority of the agents prefer $b$ to $a$ and $b$ to $c$ ). However, $f^{r}(P)=a$ for all $r \in \Re$.

Next we show that runoff SCFs are not strategy-proof.

Proposition 2 There is no runoff voting system $r \in \Re$ such that $f^{r}$ is strategy-proof.

Proof Let $n=9$. Let $P \in \mathbb{P}^{n}$ be such that there are three voters with preferences $P^{a}$, two voters with preferences $P^{b c}$, and four voters with preferences $P^{c}$. Then, $f^{r}(P)=c$ for all $r \in \Re$. Let $i \in N$ be one of the voters of type $P^{a}$ (i.e., $P_{i}=P^{a}$ ) and let $\hat{P}_{i}=P^{b c}$. Then, for all $r \in \Re, b=f^{r}\left(\hat{P}_{i}, P_{-i}\right) P_{i} f^{r}(P)=c$, which contradicts strategy-proofness.

Finally, we show that runoff SCFs are not Maskin-monotonic.

Proposition 3 There is no runoff voting system $r \in \Re$ such that $f^{r}$ is Maskinmonotonic.

Proof Consider Example 1 of the previous section. We have that $f^{r}(P)=a$ for all $r \in \Re$. Let $\hat{P} \in \mathbb{P}^{n}$ be such that there are four voters with preferences $P^{a}$, two voters with preferences $P^{b a}$, two voters with preferences $P^{b c}$ and two voters with preferences $P^{c}$ (i.e., the only difference between $P$ and $\hat{P}$ is that two voters change their preferences from $P^{c}$ to $\left.P^{b c}\right)$. Then, $f^{r}(\hat{P})=b$ for all $r \in \Re$. However, for all $i \in N$ and $x \in X$ such that $a P_{i} x$, we have $a \hat{P}_{i} x$, which contradicts Maskin-monotonicity.

\subsection{Runoff mechanisms}

Since the mechanisms in $\mathbb{G}(\Re)$ induce dynamic games of complete information, we will consider subgame perfect Nash equilibria (SPE). ${ }^{12}$

\footnotetext{
12 For each extensive form mechanism and each preference profile, a subgame perfect equilibrium induces a Nash equilibrium in every subgame (see Moore and Repullo 1988; Abreu and Sen 1990).
} 
A common problem of many voting systems is that, in the mechanisms induced by them, any alternative can be supported as an equilibrium outcome. For example, let $P \in \mathbb{P}^{n}$ be such that $P_{i}=P^{a}$ for all $i \in N$; i.e., all agents have the same preferences $a P_{i} b P_{i} c$. Given any runoff mechanism, $G \in \mathbb{G}(\Re)$, one can always find a profile of strategies that are a SPE of the mechanism at $P$ where all agents vote for alternative $c$ in Stage $1 .{ }^{13}$ Obviously, such profile of SPE strategies results in $c$.

Not all SPE are equally appealing. For instance, in Stage 1 of the previous example the agents are voting for their worst alternative $c$. This implies that they are using a dominated strategy. ${ }^{14}$ In particular, in that example, no matter what the other agents do, each agent $i$ would be at least as well voting for $a$ than voting for $c$ in Stage 1, and for some announcements of the other agents, $i$ would be strictly better announcing $a$ than announcing $c$. Because of the previous comment, we will only consider undominated SPE. ${ }^{15}$

The restriction to undominated SPE prevents from some equilibrium outcomes. This, however, does not avoid the possibility of supporting as equilibrium outcomes some alternatives which are not Condorcet winners at some preference profiles, which implies that this mechanism does not implement any Condorcet consistent SCF.

Proposition 4 Let $f: \mathbb{P}^{n} \rightarrow X$ be a Condorcet consistent SCF. Let $G \in \mathbb{G}(\Re)$ be a runoff mechanism. Then, $G$ does not implement $f$ in undominated SPE.

Proof Let $P \in \mathbb{P}^{n}$ be such that $P_{i}=P^{a}$ for all $i \in N$ (i.e., $a P_{i} b P_{i} c$ for all $i \in N$ ). Given a mechanism $G \in \mathbb{G}(\Re)$, consider a profile of strategies of that mechanism such that, for each $i \in N$ : (1) in Stage 1 agent $i$ votes for his second best alternative, $b$, and (2) in every proper subgame beginning in Stage 2, (2.1) if $a$ and $b$ are faced off against each other, agent $i$ votes for $a,(2.2)$ if $a$ and $c$ are faced off against each other, agent $i$ votes for $a$, and (2.3) if $b$ and $c$ are faced off against each other, agent $i$ votes for $b$.

We show that, for any mechanism $G \in \mathbb{G}(\Re)$, we can always find a profile of strategies for the agents satisfying the two previous conditions that constitutes an undominated SPE of $G$ at $P$.

Of course, Points (1) and (2) do not completely specify the strategy for agent $i$. The complete description of the strategy must include the actions of the agent in those contingencies that change with the particular mechanism $G \in \mathbb{G}(\Re)$ considered (i.e., contingencies that change with the particular runoff voting system considered).

\footnotetext{
13 Since the profile of strategies is a SPE, it must be such that in every proper subgame beginning at Stage 2 each agent votes for his most preferred alternative $x$ or $y$. We have not specified the complete profile of strategies since the rest of contingencies will depend on the particular mechanism. In any case, for each mechanism $M \in \mathbb{M}(\Re)$, one can always define the rest of the strategy profile in such a way that it is a SPE. In particular, the proposed profile is a Nash equilibrium of the whole game because $c$ is selected in Stage 1 and no unilateral deviation can change this result.

14 A strategy is dominated if no matter what the rest of agents do, there is some other strategy that does equally well and sometimes strictly better.

15 A profile of strategies is an undominated SPE if it is a SPE and no strategy is dominated at any subgame. A mechanism $M \in \mathbb{M}(\Re)$ implements a SCF $f$ in undominated SPE if, for each $P \in \mathbb{P}^{n}$, (i) there exists an undominated subgame perfect equilibrium of the mechanism that results in $f(P)$, and (ii) there does not exist any undominated subgame perfect equilibrium that results in some alternative different from $f(P)$.
} 
Given a mechanism $G \in \mathbb{G}(\Re)$, the complete description of the strategy can always be specified in such a way that the resulting profile of strategies constitutes a Nash equilibrium at $P$ in every subgame different from the whole game and from those subgames beginning in Stage 2, and no strategy is weakly dominated in any of these subgames at $P$.

Hence, it only remains to prove that any profile of strategies satisfying Points (1) and (2) above is such that, in the game induced by $G$ at $P$ : (i) it is a Nash equilibrium of the whole game, (ii) it induces a Nash equilibrium in every subgame that begins in Stage 2, (iii) no strategy is weakly dominated in the whole game, and (iv) no strategy is dominated in any subgame that begins in Stage 2 .

The fact that any profile of strategies satisfying Points (1) and (2) is a Nash equilibrium of the whole game is clear: since all agents are voting for $b$, this alternative is selected in Stage 1 and no unilateral deviation can change this result. The fact that such profile of strategies induces a Nash equilibrium in every subgame that begins in Stage 2 and no strategy is dominated in any of these subgames is also clear: in these subgames the agents only can vote for one of two alternatives and all of them vote for the same alternative, namely the one which they prefer most.

Finally, we prove that any strategy satisfying Points (1) and (2) is not weakly dominated in the whole game.

Claim 1 If $P_{i}=P^{a}$, a strategy where $i$ votes for $a$ in Stage 1 never dominates in the whole game a strategy satisfying Points (1) and (2).

For sake of simplicity, let us suppose that $n=9$. Let $P \in \mathbb{P}^{n}$ be such that there are three voters with preferences $P^{a}$ (agent $i$ is included in this group), two voters with preferences $P^{b c}$, and four voters with preferences $P^{c}$. Suppose that the strategy of any agent $j \neq i$ is such that, (i) in Stage 1 he votes for his most preferred alternative and, (ii) in any proper subgame beginning in Stage 2, between the two alternatives that are faced off, he votes for the one he prefers most. Suppose first that agent $i$ 's strategy is such that he votes for $a$ in Stage 1. Then three agents vote for $a$, two agents vote for $b$, and four agents vote for $c$ in Stage 1. Hence, we move to Stage 2 where alternatives $a$ and $c$ are faced off against each other and, given the strategies for all agents other than $i$, alternative $c$ is chosen, no matters what $i$ does (since the two voters with preferences $P^{b c}$ and the four voters with preferences $P^{c}$ vote for $c$ ). Suppose now that agent $i$ 's strategy satisfies Points (1) and (2). Then, $i$ votes for $b$ in Stage 1. Then, two agents vote for $a$, three agents vote for $b$, and four agents vote for $c$ in Stage 1 . Hence, we move to Stage 2 where alternatives $b$ and $c$ are faced off against each other and, given the strategies of all agents, alternative $b$ is chosen (five agents vote for $b$ and four agents vote for $c$ ). Since $b P_{i} c$, the proposed strategies for all $j \neq i$ are such that agent $i$ is better off following a strategy that satisfies Points (1) and (2) than following any strategy where he votes for $a$ in Stage 1 .

Claim 2 If $P_{i}=P^{a}$, a strategy where $i$ votes for $c$ in Stage 1 never dominates in the whole game a strategy satisfying Points (1) and (2).

This claim follows from the fact that $c$ is the worst alternative for agent $i$. We omit its proof in the interest of brevity. 
The fact that there exists a profile of strategies satisfying Points (1) and (2) that constitutes an undominated SPE of $G$ at $P$ implies that $G$ does not implement $f$ in undominated SPE, since that profile will result in $b$, which is not a Condorcet winner at $P$.

Bag et al. (2009) prove a similar result for the case in which the preferences are not restricted to be single-peaked. Since our domain of preferences is smaller, Proposition 4 cannot be deduced from their result. As in Bag et al. (2009), here the violation of Condorcet consitency is mostly due to the possible elimination of more than one alternative in the first round.

The bad properties of runoff mechanisms are beyond not delivering the Condorcet winner. They also violate Pareto-efficiency. To see this, note that the preference profile $P$ proposed in the proof of Proposition 4 is such that, even although there is an alternative, $a$, that is unanimously preferred by all agents to any other alternative, the second best alternative, $b$, can be supported as an undominated SPE. Therefore, the SCFs that are actually implemented by the runoff mechanisms violate unanimity and, hence, are not Pareto-efficient either. ${ }^{16}$

The fact that runoff voting systems do not implement Condorcet consistent SCFs in undominated SPE does not imply that Condorcet consistent SCFs are not implementable. Our next proposition shows that some Condorcet consistent SCFs can be implemented in dominant strategies via simple mechanisms.

For each $P \in \mathbb{P}^{n}$, an alternative $x \in X$ is a median of the peaks if $\#\{i \in N$ : $\left.p\left(P_{i}\right) \geq x\right\} \geq \frac{n}{2}$ and $\#\left\{i \in N: x \geq p\left(P_{i}\right)\right\} \geq \frac{n}{2}$ (given the order $\left.a>b>c\right) .{ }^{17}$ Since in our model, preferences are restricted to be single-peaked relative to the order $a>b>c$, the median of the peaks relative to this order are the Condorcet winners. In our context, the median of the peaks may not be unique. This implies that there are several Condorcet consistent SCFs. We prove that some of them are implementable in dominant strategies via a simple mechanism.

Before showing this result, let us note that, if there are more than one median, alternative $b$ must be one of them. In other words, the possible sets of median of the peaks are $\{a\},\{b\},\{c\},\{a, b\},\{b, c\}$, and $\{a, b, c\}$. For each $P \in \mathbb{P}^{n}$, let $\operatorname{med}(P)$ be the set of the median of the peaks. Let $f^{*}: \mathbb{P}^{n} \rightarrow X$ be the Condorcet consistent SCF that selects $b$ when there are several Condorcet winners; i.e., for each $P \in \mathbb{P}^{n}$,

$$
f^{*}(P)= \begin{cases}\operatorname{med}(P) ; & \text { if } \operatorname{med}(P) \text { is unique } \\ b ; & \text { otherwise }\end{cases}
$$

Let $\Gamma^{*}=(M, g)$ be the normal form mechanism such that:

\footnotetext{
16 Runoff mechanisms have at least one good property: no Condorcet loser can be sustained as an equilibrium (an alternative is a Condorcet loser if can be defeated in pairwise comparisons by any other alternative).

17 For example, if $n=10$ and $P \in \mathbb{P}^{n}$ is such that five agents have their peaks in $a$, one agent has his peak in $b$, and four agents have their peaks in $c$, then both $a$ and $b$ are medians of the peaks. If $\hat{P} \in \mathbb{P}^{n}$ is such that five agents have their peaks in $a$ and five agents have their peaks in $c$, then all the alternatives, $a$, $b$, and $c$, are medians of the peaks.
} 
- For each $i \in N, M_{i}=\{a, b, c\}$; i.e., each agent only has to announce one alternative. For each $m \in M$, let $\operatorname{med}(m)$ be the set of the median of the announcements $\left\{m_{1}, m_{2}, \ldots, m_{n}\right\}$ relative to the order $a>b>c .^{18}$

- The outcome function $g: M \rightarrow X$ is such that, for each $m \in M$ :

$$
g(m)= \begin{cases}\operatorname{med}(m) ; & \text { if } \operatorname{med}(m) \text { is unique } \\ b ; & \text { otherwise }\end{cases}
$$

Mechanism $\Gamma^{*}$ is a simplified version of the direct mechanism associated with $f^{*}$ where each agent only has to announce his most preferred alternative (instead of having to announce his whole preference relation).

Proposition 5 Mechanism $\Gamma^{*}$ implements in dominant strategies the Condorcet consistent $S C F f^{*}$.

Proof Claim 1 For each $P \in \mathbb{P}^{n}$ and $i \in N, m_{i}=p\left(P_{i}\right)$ is a dominant strategy of $\Gamma^{*}$ at $P$ for agent $i$.

Case 1.1 Suppose that $p\left(P_{i}\right)=a$. Then $P_{i}=P^{a}$. Suppose by contradiction that $m_{i}=a$ is not a dominant strategy for $i$. Then, there exists some $\hat{m}_{i} \neq a$ and some $\hat{m}_{-i} \in M_{-i}$ such that $g\left(\hat{m}_{i}, \hat{m}_{-i}\right) P^{a} g\left(a, \hat{m}_{-i}\right)$. Since $a P^{a} b P^{a} c$, we have that either $g\left(a, \hat{m}_{-i}\right)=b$ or $g\left(a, \hat{m}_{-i}\right)=c$. If $g\left(a, \hat{m}_{-i}\right)=b$, then $g\left(\tilde{m}_{i}, \hat{m}_{-i}\right) \neq a$ for all $\tilde{m}_{i} \in M_{i}$, and therefore $g\left(a, \hat{m}_{-i}\right) R^{a} g\left(\hat{m}_{i}, \hat{m}_{-i}\right)$. If $g\left(a, \hat{m}_{-i}\right)=c$, then $g\left(\tilde{m}_{i}, \hat{m}_{-i}\right)=c$ for all $\tilde{m}_{i} \in M_{i}$, and therefore $g\left(a, \hat{m}_{-i}\right) P^{a} g\left(\hat{m}_{i}, \hat{m}_{-i}\right)$.

Case 1.2 Suppose that $p\left(P_{i}\right)=b$. Then either $P_{i}=P^{b a}$ or $P_{i}=P^{b c}$. Suppose by contradiction that there exists some $\hat{m}_{i} \neq b$ and some $\hat{m}_{-i} \in M_{-i}$ such that $g\left(\hat{m}_{i}, \hat{m}_{-i}\right) P^{b a} g\left(b, \hat{m}_{-i}\right)$ or $g\left(\hat{m}_{i}, \hat{m}_{-i}\right) P^{b c} g\left(b, \hat{m}_{-i}\right)$. Since $b P^{b a} a P^{b a} c$ and $b$ $P^{b c} c P^{b c} a$, we have that either $g\left(b, \hat{m}_{-i}\right)=a$ or $g\left(b, \hat{m}_{-i}\right)=c$. If $g\left(b, \hat{m}_{-i}\right)=a$ then $g\left(\tilde{m}_{i}, \hat{m}_{-i}\right)=a$ for all $\tilde{m}_{i} \in M_{i}$, and therefore $g\left(b, \hat{m}_{-i}\right) R^{b a} g\left(\hat{m}_{i}, \hat{m}_{-i}\right)$ and $g\left(b, \hat{m}_{-i}\right) R^{b c} g\left(\hat{m}_{i}, \hat{m}_{-i}\right)$. If $g\left(b, \hat{m}_{-i}\right)=c$ then $g\left(\tilde{m}_{i}, \hat{m}_{-i}\right)=c$ for all $\tilde{m}_{i} \in M_{i}$, and therefore $g\left(b, \hat{m}_{-i}\right) R^{b a} g\left(\hat{m}_{i}, \hat{m}_{-i}\right)$ and $g\left(b, \hat{m}_{-i}\right) R^{b c} g\left(\hat{m}_{i}, \hat{m}_{-i}\right)$.

Case 1.3 Suppose that $p\left(P_{i}\right)=c$. Then $P_{i}=P^{c}$. Suppose by contradiction that there exists some $\hat{m}_{i} \neq c$ and some $\hat{m}_{-i} \in M_{-i}$ such that $g\left(\hat{m}_{i}, \hat{m}_{-i}\right) P^{c} g\left(c, \hat{m}_{-i}\right)$. Since $c P^{c} b P^{c} a$, we have that either $g\left(c, \hat{m}_{-i}\right)=b$ or $g\left(c, \hat{m}_{-i}\right)=a$. If $g\left(c, \hat{m}_{-i}\right)=b$, then $g\left(\tilde{m}_{i}, \hat{m}_{-i}\right) \neq c$ for all $\tilde{m}_{i} \in M_{i}$, and therefore $g\left(c, \hat{m}_{-i}\right) R^{c} g\left(\hat{m}_{i}, \hat{m}_{-i}\right)$. If $g\left(c, \hat{m}_{-i}\right)=a$, then $g\left(\tilde{m}_{i}, \hat{m}_{-i}\right)=a$ for all $\tilde{m}_{i} \in M_{i}$, and therefore $g\left(c, \hat{m}_{-i}\right) P^{c}$ $g\left(\hat{m}_{i}, \hat{m}_{-i}\right)$.

Claim 2 For each $P \in \mathbb{P}^{n}, i \in N$, and $m_{i} \neq p\left(P_{i}\right)$, we have that $m_{i}$ is not a dominant strategy of $\Gamma^{*}$ at $P$ for agent $i$.

Suppose for simplicity that $n=10$.

\footnotetext{
18 That is, $\operatorname{med}(m)$ is the median of the peaks when the preference profile $P \in \mathbb{P}^{n}$ is such that $p\left(P_{i}\right)=m_{i}$ for all $i \in N$.
} 
Case 2.1 Suppose that $p\left(P_{i}\right)=a$. Then $P_{i}=P^{a}$. Let $m_{-i} \in M_{-i}$ be such that five agents announce $a$ and four agents announce $b$. Then, $a=g\left(a, m_{-i}\right) P^{a} g\left(b, m_{-i}\right)=$ $b$, and hence $m_{i}=b$ is not a dominant strategy of $\Gamma^{*}$ at $P$ for agent $i$. Similarly, let $\hat{m}_{-i} \in M_{-i}$ be such that four agents announce $b$ and five agents announce $c$. Then, $b=g\left(a, \hat{m}_{-i}\right) P^{a} g\left(c, \hat{m}_{-i}\right)=c$, and hence $m_{i}=c$ is not a dominant strategy of $\Gamma^{*}$ at $P$ for agent $i$.

Case 2.2 Suppose that $p\left(P_{i}\right)=b$. Then either $P_{i}=P^{b a}$ or $P_{i}=P^{b c}$. Let $m_{-i} \in$ $M_{-i}$ be such that five agents announce $a$ and four agents announce $b$. Then, $b=$ $g\left(b, m_{-i}\right) P_{i} g\left(a, m_{-i}\right)=a$, and hence $m_{i}=a$ is not a dominant strategy of $\Gamma^{*}$ at $P$ for agent $i$. Similarly, let $\hat{m}_{-i} \in M_{-i}$ be such that four agents announce $b$ and five agents announce $c$. Then, $b=g\left(b, \hat{m}_{-i}\right) P_{i} g\left(c, \hat{m}_{-i}\right)=c$, and hence $m_{i}=c$ is not a dominant strategy of $\Gamma^{*}$ at $P$ for agent $i$.

Case 2.3 Suppose that $p\left(P_{i}\right)=c$. Then $P_{i}=P^{c}$. Let $m_{-i} \in M_{-i}$ be such that five agents announce $a$ and four agents announce $b$. Then, $b=g\left(c, m_{-i}\right) P^{c} g\left(a, m_{-i}\right)=$ $a$, and hence $m_{i}=a$ is not a dominant strategy of $\Gamma^{*}$ at $P$ for agent $i$. Similarly, let $\hat{m}_{-i} \in M_{-i}$ be such that four agents announce $b$ and five agents announce $c$. Then, $c=g\left(c, \hat{m}_{-i}\right) P^{c} g\left(b, \hat{m}_{-i}\right)=b$, and hence $m_{i}=b$ is not a dominant strategy of $\Gamma^{*}$ at $P$ for agent $i$.

Not every Condorcet consistent SCF is implementable in dominant strategies. The following example clarifies this point.

Example 2 Consider the SCF $\hat{f}: \mathbb{P}^{n} \rightarrow X$ such that, for each $P \in \mathbb{P}^{n}$,

$$
\hat{f}(P)= \begin{cases}\operatorname{med}(P) ; & \text { if } \operatorname{med}(P) \text { is unique } \\ a ; & \text { if } \operatorname{med}(P)=\{a, b\} \\ c ; & \text { if } \operatorname{med}(P)=\{b, c\} \\ b ; & \text { if } \operatorname{med}(P)=\{a, b, c\}\end{cases}
$$

Clearly, $\hat{f}$ is Condorcet consistent. Suppose that $n=10$. Let $P \in \mathbb{P}^{n}$ be such that five agents have preferences $P^{a}$, one agent has preferences $P^{b a}$, and four agents have preferences $P^{c}$. Let $i \in N$ be the agent whose preferences are $P^{b a}$. Note that $b=\hat{f}\left(P^{c}, P_{-i}\right) P^{b a} \hat{f}\left(P^{b a}, P_{-i}\right)=a$. In other words, the SCF $\hat{f}$ is not strategyproof. Hence, $\hat{f}$ is not implementable in dominant strategies (via a mechanism similar to $\Gamma^{*}$ or via any other mechanism).

\section{Conclusion}

The Constitutional Court of Spain uses runoff as voting system to decide whether a law is constitutional, unconstitutional, or interpretable. It is known that, for universal domains of preferences, runoff voting systems have several drawbacks as social choice rules. In the setting of this paper, however, the preferences of the judges are restricted to be single-peaked over three alternatives (i.e., if the most preferred alternative for a 
judge is to declare the law constitutional, then to declare the law unconstitutional is his less preferred alternative, and vice versa). We show that, unfortunately, this restriction on the domain is not enough to solve the problems of the runoff social choice rules. Alternatively, runoff voting systems can be analyzed as mechanisms instead of as social choice rules. Since in our setting there always exist Condorcet winners, one question that arises naturally if we follow this approach is whether the runoff mechanisms implement Condorcet consistent social choice rules, and whether there are other mechanisms that can make this job better. We prove that runoff mechanisms are not well-behaved: we show that they do not implement Condorcet consistent social choice rules in undominated subgame perfect Nash equilibria. We show, however, that some Condorcet consistent functions can be implemented in dominant strategies via a simple and natural mechanism in which each judge only has to announce one of the three possible resolutions: constitutional, interpretable, or unconstitutional. Thus, this mechanism overtakes the runoff mechanism in terms of the majoritarian approval. We conclude, therefore, that the rules governing courts deserve more detailed analysis as some of its procedures may fail at achieving the will of the majority of the judges.

We see some scope for further development and extension of the model studied in this paper. In our model, the judges know perfectly well their preferences. We interpret the situation as follows: The voting procedure of the constitutional court is a way to aggregate judges' criteria. Such criteria (which are simply personal opinions based on experiences, knowledge, and how each judge understands and interprets the constitution) may differ across judges. A different line of research could involve assuming that there is an objective true about the law's constitutionality that the judges are trying to uncover. This approach is related to de literature dealing with the Condorcet jury theorem (e.g., AustenSmith and Banks 1996; Feddersen and Pesendorfer 1998; Duggan and Martinelli 2001).

Acknowledgments We thank Luis Corchón and two anonymous referees for their helpful comments. Financial assistance from Ministerio de Educación y Ciencia under project ECO2008-03674/ECON is gratefully acknowledged.

Open Access This article is distributed under the terms of the Creative Commons Attribution License which permits any use, distribution and reproduction in any medium, provided the original author(s) and source are credited.

\section{References}

Abreu D, Sen A (1990) Subgame perfect implementation: A necessary and sufficient condition. J Econ Theory 50:285-299

Austen-Smith D, Banks JS (1996) Information aggregation, rationality and the Condorcet jury theorem. Amer Polit Sci Rev 90:34-45

Barberà S (2010) Strategy-proof social choice, In: Arrow K, Suzumura K (eds) Handbook of social choice and welfare. vol 2. Kluwer Academic Publishers, Dordrecht (forthcoming)

Bag PK, Sabourian H, Winter E (2009) Multi-stage voting, sequential elimination and Condorcet consistency. J Econ Theory 144:1278-1299 
Duggan J, Martinelli C (2001) A Bayesian model of voting in juries. Games Econ Behav 37:259-294

Feddersen TJ, Pesendorfer W (1998) Convicting the innocent: The inferiority of unanimous jury verdict. Amer Polit Sci Rev 92:23-35

Fishburn PC, Brams SJ (1981) Aproval voting, Condorcet's principle, and runoff elections. Public Choice 36:89-114

Gibbard A (1973) Manipulation of voting schemes: A general result. Econometrica 41:587-601

Grofman B (2008) A taxonomy of runoff methods. Elect Stud 27:395-399

Maskin E (1999) Nash equilibrium and welfare optimality. Rev Econ Stud 66:23-38

Moore J, Repullo R (1988) Subgame perfect implementation. Econometrica 56:1191-1220

Moulin H (1988) Axioms of cooperative decision making. Cambridge University Press, Cambridge 